\title{
The Influence of Autologous Bone Marrow Stem Cell Transplantation on Matrix Metalloproteinases in Patients Treated for Acute ST-Elevation Myocardial Infarction
}

\author{
Eline Bredal Furenes, ${ }^{1,2,3}$ Trine Baur Opstad, ${ }^{1,2,3}$ Svein Solheim, ${ }^{1,2}$ \\ Ketil Lunde, ${ }^{4}$ Harald Arnesen, ${ }^{1,2,3}$ and Ingebjørg Seljeflot ${ }^{1,2,3}$ \\ ${ }^{1}$ Center for Clinical Heart Research, Department of Cardiology, Oslo University Hospital Ullevål, Postboks 4956, \\ Nydalen, 0424 Oslo, Norway \\ ${ }^{2}$ Center for Heart Failure Research, Institute for Experimental Medical Research, Ullevål University Hospital, \\ Kirkeveien 166, 0407 Oslo, Norway \\ ${ }^{3}$ Faculty of Medicine, University of Oslo, Postboks 1078, Blindern, 0316 Oslo, Norway \\ ${ }^{4}$ Department of Cardiology, Oslo University Hospital, National Hospital, Postboks 4950, Nydalen, 0424 Oslo, Norway
}

Correspondence should be addressed to Eline Bredal Furenes; furenes1@gmail.com

Received 5 June 2014; Revised 11 August 2014; Accepted 27 August 2014; Published 11 September 2014

Academic Editor: José Cesar Rosa Neto

Copyright (C) 2014 Eline Bredal Furenes et al. This is an open access article distributed under the Creative Commons Attribution License, which permits unrestricted use, distribution, and reproduction in any medium, provided the original work is properly cited.

\begin{abstract}
Background. Matrix metalloproteinase-9 (MMP-9), regulated by tissue inhibitor of metalloproteinase-9 (TIMP-1) and the extracellular matrix metalloproteinase inducer (EMMPRIN), contributes to plaque instability. Autologous stem cells from bone marrow (mBMC) treatment are suggested to reduce myocardial damage; however, limited data exists on the influence of mBMC on MMPs. Aim. We investigated the influence of mBMC on circulating levels of MMP-9, TIMP-1, and EMMPRIN at different time points in patients included in the randomized Autologous Stem-Cell Transplantation in Acute Myocardial Infarction (ASTAMI) trial $(n=100)$. Gene expression analyses were additionally performed. Results. After 2-3 weeks we observed a more pronounced increase in MMP-9 levels in the mBMC group, compared to controls $(P=0.030)$, whereas EMMPRIN levels were reduced from baseline to $2-3$ weeks and 3 months in both groups $(P<0.0001)$. Gene expression of both MMP-9 and EMMPRIN was reduced from baseline to 3 months. MMP-9 and EMMPRIN were significantly correlated to myocardial injury (CK: $P=0.005$ and $P<0.001$, resp.) and infarct size (SPECT: $P=0.018$ and $P=0.008$, resp.). Conclusion. The results indicate that the regulation of metalloproteinases is important during AMI, however, limited influenced by mBMC.
\end{abstract}

\section{Introduction}

The early and late mortality after acute myocardial infarction (AMI) is declining, but cardiovascular disease (CVD) is still one of the leading causes of morbidity and death in the Western world. Ischemic heart disease is a feared, but often inevitable complication of atherosclerosis, the main underlying cause of myocardial infarction $[1,2]$. Inflammation is considered to be a key process for development of atherosclerosis and this includes a number of cellular and molecular responses resulting in plaque formation $[2,3]$. Despite well-documented treatment of AMI survivors, both medically and by percutaneous coronary intervention (PCI), some patients either do not receive this treatment, do not respond satisfactorily, or develop congestive heart failure despite treatment.

Several animal studies have shown that bone marrow stem cells differentiate to cardiomyocytes when infused into the affected myocardium [4]. Treatment with autologous stem cells from bone marrow has been suggested to reduce myocardial damage in patients with AMI. Results from clinical trials are, however, conflicting with regard to improvement of left ventricular ejection fraction [5-10]. Possible mechanisms by which autologous bone marrow stem cells 
act are discussed to be cardiac transdifferentiation, paracrine effects, angiogenesis, and reduced apoptosis [11, 12].

Matrix metalloproteinases (MMPs), a class of 24 endopeptidases, participate in plaque instability by degrading the extracellular matrix. MMP-9, a zinc-dependent gelatinase, is found in the shoulder of the plaque, contributes to plack instability and rupture, and has been associated with acute coronary syndrome (ACS) $[3,13]$. Circulating MMP-9 has been shown to be elevated in patients with AMI, stable, and unstable angina pectoris $[13,14]$, as well as in hypertensives [15] and smokers [16]. In addition, MMP-9 is discussed to be involved in adverse left ventricular remodelling and associated with higher cardiovascular risk score [13]. The MMPs are regulated by specific endogenous inhibitors, tissue inhibitor of metalloproteinases (TIMPs), and MMP-9 is specifically regulated when pro-MMP-9 binds to TIMP-1 $[14,17]$.

Lately, the extracellular matrix metalloproteinase inducer (EMMPRIN, CD147), a member of the immunoglobulin superfamily, has been discussed to be involved in both expression and release of MMP-9 [18], thus, having a potentially regulatory role in CVD. EMMPRIN has been shown to be expressed in atherosclerotic plaques [19] as well as in cell types like monocytes, macrophages, and platelets [20].

In humans, limited data exists on the influence of bone marrow stem cells on MMPs that may be of importance for the myocardium and the infarction process. In the "randomized Autologous Stem-Cell Transplantation in Acute Myocardial Infarction" (ASTAMI) trial [10], the main aim was to assess the effects of intracoronary injection of autologous mononuclear bone marrow-derived cells (mBMC) on left ventricular ejection fraction in patients with ST-elevation myocardial infarction (STEMI). The main hypothesis was that the treatment would reduce the infarction sequelae, which, however, could not be demonstrated [10].

The aim of this ASTAMI substudy was to investigate the influence of coronary injection of mBMC on MMP-9, TIMP-1, and EMMPRIN, circulating levels as well as on gene expression in leukocytes, in patients with STEMI undergoing successful PCI. Furthermore, to investigate any association between the measured biomarkers and infarct size and left ventricular function, our hypothesis was that MMP-9 levels would be reduced after $\mathrm{mBMC}$ treatment, in parallel with reduction of EMMPRIN.

\section{Materials and Methods}

2.1. Subjects and Study Design. The ASTAMI study design and treatment protocol have previously been described in details [21]. Briefly, it was a randomized 1:1 open-labelled study, where one arm was intracoronary treatment with $\mathrm{mBMC}$, and the other controls without aspiration and injection of bone marrow. Patients, age between 40 and 75 years, both gender were included. They were all treated with PCI with stent implantation in the left anterior descending (LAD) coronary artery.

Exclusion criteria were previous Q-wave infarction, cardiogenic shock, or severe comorbidity interfering with compliance to the protocol.
Baseline recordings were performed during day 4-5 after AMI, before bone marrow aspiration (in the treatment group) [10].

The study protocol, including the biobank, was approved by the Regional Committee for Medical Research Ethics and all patients gave written, informed consent. The study is registered at ClinicalTrials.gov, NCT 00199823.

\subsection{Laboratory Methods}

2.2.1. Blood Sampling. A biobank, kept at $-80^{\circ} \mathrm{C}$ consisting of plasma, serum, and PaxGene tubes (PreAnalytiX GmbH, Hombrechtikon, $\mathrm{CH}$ ), the latter for gene expression measures in circulating leukocytes, was established. Blood samples were collected in fasting condition between 08.00 and 10.00 am the day before transplantation in the $\mathrm{mBMC}$ group (day1) (baseline), the day after (day 1) and further day 3, after 2-3 weeks and after 3 months. The same time interval was used for the control group, except baseline sampling (day-1) which was drawn median 4 days after PCI compared to 5 days in the mBMC group.

2.2.2. Enzyme Immunoassays. For analyses of MMP-9, TIMP-1, and EMMPRIN commercial enzyme linked immunosorbent assays (ELISA) (R\&D Systems Europe, Abingdon, Oxford, UK) were used on serum samples, which were performed within 1 hour by centrifugation at room temperature $2500 \times \mathrm{g}$ for $10 \mathrm{~min}$. The interassay coefficients of variation $(\mathrm{CV})$ were $7.3 \%$ for MMP-9, 4.4\% for TIMP-1, and $5.4 \%$ for EMMPRIN.

2.2.3. Gene Expression Analysis. Isolation of RNA from PaxGene tubes was performed according to the manufacturers instruction (PreAnalytix, Qiagen $\mathrm{GmbH}$, Germany) in a subset of randomly selected samples $(n=47)$, with an additional cleaning step (Rneasy MinElute Cleanup Kit, Qiagen). A complementary DNA (cDNA) of the messenger RNA (mRNA) content was achieved by inversely transcribing total RNA in the samples. The genetic expression of mRNA of MMP-9 and EMMPRIN was performed by use of realtime PCR on the ViiA 7 Real Time PCR System (Applied Biosystems, Foster City, CA, USA) and the $\Delta \Delta \mathrm{Ct}$ method was applied [22]. This relative or comparative Ct method determines the relative target quantity (RQ-values) in the samples, by measuring the amplification (crossing threshold $\mathrm{Ct}$ ) of the target samples and in a reference sample and normalized to an endogenous control (house-keeping gene). Assays for the target genes were Hs00234579_ml for MMP9, Hs00936295_ml for EMMPRIN, and $\beta-2$ macroglobulin (Hs99999907_m1) was chosen as house-keeping gene.

2.2.4. Measures of Myocardial Function. Left ventricular ejection fraction (LVEF) and infarct size were obtained by electrocardiogram-gated single photon emission computed tomography (SPECT) (GE Medical Systems with 4DMSPECT software) at baseline $(4.0 \pm 1.4$ days after the AMI). Infarct size was expressed as percentage of the LAD-area. 
TABLE 1: Characteristics of the study population according to the randomized groups. Values are presented as proportion, means \pm SD, or medians ${ }^{1}$ with 25 th and 75 th percentiles.

\begin{tabular}{|c|c|c|}
\hline & mBMC group $(n=50)$ & Control group $(n=50)$ \\
\hline Age (years) & $58.1(8.6)$ & $56.7(9.6)$ \\
\hline Gender ( $\%$ female) & 16 & 16 \\
\hline Hypertension (\%) & 35 & 34 \\
\hline Diabetes (\%) & 10 & 8 \\
\hline Smokers (\%) & 39 & 48 \\
\hline BMI $\left(\mathrm{kg} / \mathrm{m}^{2}\right)$ & $26.3(3.3)$ & $27.1(4.1)$ \\
\hline SBP/DBP (mmHg) & $131 / 82 \pm 21 / 14$ & $132 / 83 \pm 23 / 17$ \\
\hline Tot. chol (mmol/L) & $4.4 \pm 0.9$ & $4.5 \pm 0.9$ \\
\hline LDL chol (mmol/L) & $2.9 \pm 0.8$ & $2.9 \pm 0.7$ \\
\hline HDL chol (mmol/L) & $1.0 \pm 0.3$ & $1.1 \pm 0.3$ \\
\hline Triglycerides $(\mathrm{mmol} / \mathrm{L})^{1}$ & $1.3(1.0,1.7)$ & $1.3 \pm(1.1,1.5)$ \\
\hline Symptom start to PCI $(\mathrm{min})^{1}$ & $210(180,330)$ & $230(180,330)$ \\
\hline LVEF (\%) & $41.3(10.6)$ & $42.6(11.7)$ \\
\hline Infarct size (\%) & $43.7 \pm 17.6$ & $40.7 \pm 19.3$ \\
\hline Peak CK (U/L) & $3338 \pm 2398$ & $3532 \pm 2650$ \\
\hline Thrombolysis before PCI (\%) & 30 & 28 \\
\hline \multicolumn{3}{|l|}{ Medication at discharge } \\
\hline Aspirin (\%) & 100 & 100 \\
\hline Clopidogrel (\%) & 100 & 100 \\
\hline ACE-I/ATII antagonist (\%) & 100 & 100 \\
\hline$\beta$-blocker (\%) & 100 & 100 \\
\hline Diuretics (\%) & 43 & 32 \\
\hline Statin (\%) & 100 & 100 \\
\hline
\end{tabular}

ATII antagonist: angiotensin II receptor antagonist; ACE-I: angiotensin-converting enzyme inhibitor; BMI: body mass index; DBP: diastolic blood pressure; LVEF: left ventricular ejection fraction; PCI: percutaneous coronary intervention; and SBP: systolic blood pressure.

2.3. Statistical Analysis. MMP-9, TIMP-1, and EMMPRIN levels were all skewly distributed and nonparametric statistics were used throughout. Median values and 25 and 75 percentiles are given unless otherwise stated. For group comparisons the Mann-Whitney test was used for continuous data and Chi square test for categorical data. Friedman test was performed to analyze for differences between any time points within the groups. For changes from baseline (day1) to the subsequent time points, Wilcoxon test was used only when Friedman test was significant. For differences in changes between the randomized groups, Mann-Whitney test was used. Spearman's rho was used for correlation analysis. The level of significance was set to $P<0.05$. The SPSS software package version 18.0 was used throughout.

\section{Results}

Baseline characteristics of the study population according to the randomized groups are shown in Table 1.

One hundred patients included in the ASTAMI study, 50 randomized to $\mathrm{mBMC}$ treatment and 50 controls, were followed. From one patient we did not obtain blood samples. The groups did not differ regarding baseline characteristics. They were all medically treated according to current guidelines, thus, all patients were on statins, beta-blockers, ACEinhibitors/ATII antagonists, and antithrombotic agents.
3.1. Circulating Levels of MMPs (Table 2). Although blood samples at baseline were obtained one day later (median) from symptom start of AMI in the mBMC group compared to controls, no significant differences between the groups were recorded at baseline (day-1). No differences in MMP9, TIMP-1, or EMMPRIN between the mBMC group and controls were seen at any further time points.

In the $\mathrm{mBMC}$ group there was a significant increase in MMP-9 levels from baseline to 2-3 weeks $(P=0.009)$, which could not be demonstrated in the control group. We observed no within group changes in TIMP-1 levels in either groups. EMMPRIN levels were significantly reduced from baseline to $2-3$ weeks and 3 months in both groups $(P<0.0001$, all).

When analyzing for differences between the groups in changes from baseline to further time points, we could demonstrate a significant difference in change in MMP-9 levels between the two groups at 2-3 weeks and after 3 months, showing a more pronounced increase in the $\mathrm{mBMC}$ group $(P=0.030$ and $P=0.05$, resp.). No differences in changes between the groups were observed for TIMP-1 and EMMPRIN.

3.2. Gene Expression Levels. We observed no differences in MMP-9 gene expression between the two groups at any time points, while the EMMPRIN gene expression was significantly lower in the mBMC group $(n=23)$ versus the controls 


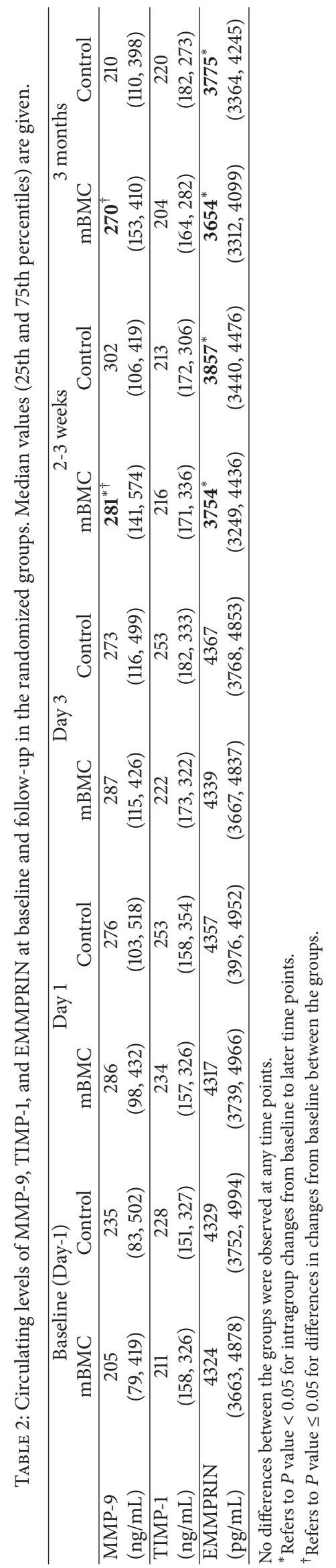


$(n=24)$ after 3 months $(P=0.03)$ (Table 3$)$. The levels of both MMP-9 and EMMPRIN gene expressions were significantly reduced from baseline to 3 months in the $\mathrm{mBMC}$ group $(P<0.0001$ and $P=0.002$, resp.). This could not be demonstrated in the control group. There were, however, no differences between the groups in changes from baseline to any later time points. When defining baseline mRNA level (RQ-values) in the total population to 1 , there was a $20 \%$ reduction in MMP-9 gene expression from baseline to 2-3 weeks, and a $50 \%$ reduction after 3 months in the mBMC group. A similar pattern was seen in gene expression of EMMPRIN, with a $20 \%$ reduction after $2-3$ weeks and $60 \%$ reduction after 3 months in the mBMC group. The results of the gene expression presented as fold changes are illustrated in Figure 1.

3.3. Correlations. In the total population at baseline we observed a significant correlation between MMP-9 and EMMPRIN $(r=0.25, P=0.011)$. A strong correlation was also shown between MMP-9 and TIMP-1 $(r=0.66$, $P<0.0001)$ and also between TIMP-1 and EMMPRIN $(r=$ $0.36, P=0.01)$. Significant correlations between $\mathrm{CK}$ and baseline levels of both MMP-9 and EMMPRIN were found ( $r=0.29 P=0.005$ and $r=0.43 P<0.001$, resp.). MMP9 and EMMPRIN, but not TIMP-1, showed also significant correlations to infarct size measured by SPECT $(r=0.24$, $P=0.018$ and $r=0.27 P=0.008$, resp.). EMMPRIN levels were also found to be inversely correlated to LVEF at baseline $(r=-0.31 P=0.002)$.

We observed no significant correlations between circulating MMP-9 and gene expression of MMP-9 at any time points in the total population, or in the single groups. Likewise there were no correlations between circulating EMMPRIN and gene expression of EMMPRIN, or between gene expression of EMMPRIN and MMP-9 levels in either groups or in the total population.

\section{Discussion}

The main finding in the present study was that there was limited influence of intracoronary injection of mBMC transplantation after AMI on circulating levels of MMP-9, TIMP-1, and EMMPRIN, other than a more pronounced increase in MMP-9 after 2-3 weeks in the mBMC group. EMMPRIN levels were reduced after 2-3 weeks and 3 months in both groups. At baseline both MMP-9 and EMMPRIN were significantly correlated to myocardial injury assessed by biomarkers and infarct size and might therefore support their predictory ability for later outcome.

All patients were medically treated according to current guidelines; thus, any influence by medication on the measured variables would be equally affected in the randomized groups.

In both in vitro and in vivo studies, stem cell transplantation has been shown to reduce MMPs after AMI and improve ventricular remodeling [23]. Our hypothesis was therefore that treatment with $\mathrm{mBMC}$ would reduce the circulating levels of the selected biomarkers. In a study using modified mesenchymal stem cell transplantation into AMI rat hearts [24], a reduction in MMP-9 levels was shown. Mesenchymal stem cells are multipotent stromal cells that can differentiate into a variety of cell types [25], and the results are thus not quite comparable to ours. In addition, stem cell injection was performed one hour after AMI. In contrast, we found a significantly more pronounced increase in MMP-9 levels from baseline to 2-3 weeks in the $\mathrm{mBMC}$ group compared to controls. In accordance with our findings Roderfelt et al. demonstrated a transient inflammatory response and upregulation of MMP-9 activity after bone marrow transplantation in $A b c b 4^{-/-}$(hepatic fibrosis) mice [26]. We have previously shown that MMP-9 levels are reduced 1 day after AMI [27]. Therefore we assume that the levels were normalized when baseline sampling in the present study was performed and limited influenced by the acute phase reaction. In the control group in our study, bone marrow aspiration was not performed. This procedure which itself is a trauma could influence the release of inflammatory markers and contribute to the elevated levels in the mBMC group.

In the study by Shu et al. using mesenchymal stem cell transplantation, TIMP-1 levels did not vary significantly [24], which is in accordance with our findings of no changes in this variable during the observation period in any of the groups.

The significant reduction in genetic expression of MMP9 seen at 3 months might be discussed as compensatory to the increase observed in the circulating levels. MMP9 expression is a crucial pathogenic feature in a range of conditions and disease states, also other than CVD [28$30]$, in which treatment with stem cells has been shown to suppress or downregulate the MMP-9 expression [30] and thereby improving the current condition.

The underlying mechanisms for the influence of stem cells on MMPs are not clarified. In cell culture of cardiac fibroblasts Wang et al. [31] could demonstrate that the protein expression and activity of MMP-2, but not MMP-9, were increased in response to hypoxia and decreased when cocultured with mesenchymal stem cells. It has also been demonstrated that early endothelial progenitor cells increased MMP-9 expression in vitro, whereas MMP-2 was increased in outgrowth endothelial cells [32]. The type of stem cells seems to be of importance regarding the degree of influence on MMPs [33].

The importance of EMMPRIN as an inducer of MMP-9 has been explored to a limited extent in humans. In our study circulating levels of MMP-9 and EMMPRIN were significantly correlated, indicating a common regulatory pathway [18]. Circulating levels as well as genetic expression of EMMPRIN were significantly reduced along with the increase in MMP-9. This might be discussed as a negative feedback mechanism. There was, however, no influence of mBMC on circulating levels or gene expression of EMMPRIN, shown by the significant reduction in both groups during the observation period. Expression of the EMMPRIN-gene in circulating leukocytes, also reported by $\mathrm{Xu}$ et al. assessed by flow cytometry [34], may indicate that the leukocytes contribute to the circulating levels, although no correlation between circulating levels and gene expression was observed. The reduction over time seen in EMMPRIN expression, with 


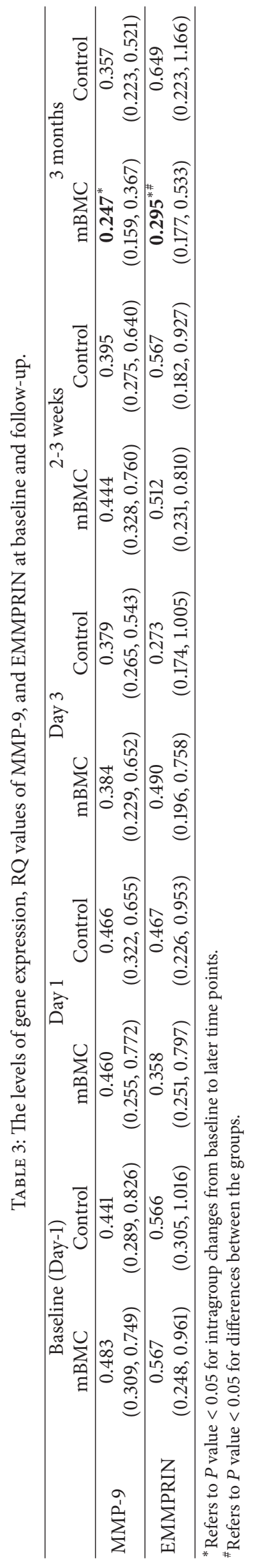




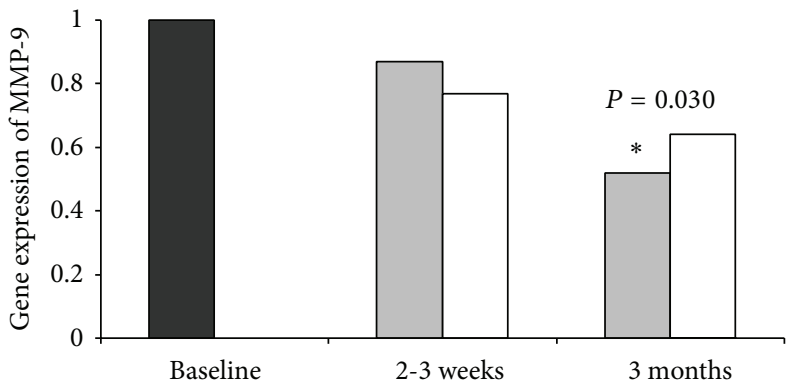

(a)

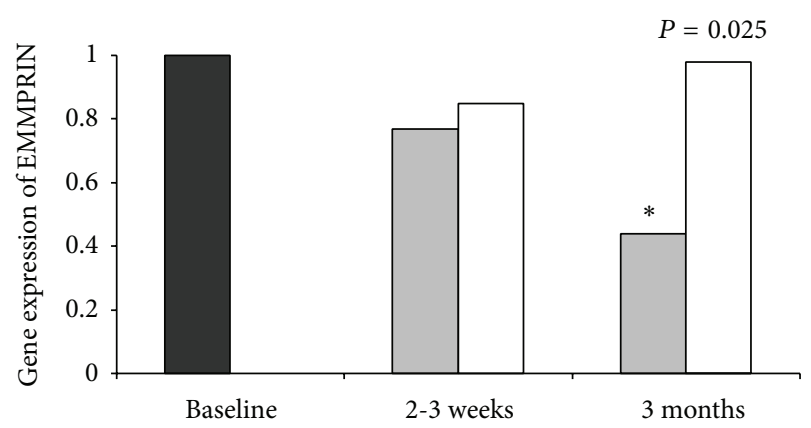

(b)

FIGURE 1: Gene expression of MMP-9 (a) and EMMPRIN (b) after 2-3 weeks and 3 months in the mBMC group and controls relative to baseline expression in the total population. Black bars: total group at baseline; grey bars: mBMC group; white bars: controls. ${ }^{*}$ indicates reduction from baseline to 3 months in the $\mathrm{mBMC}$ group $P$ values refer to differences in expression relative to baseline between groups.

subsequent reduction in MMP-9 gene expression, contributes to the assumption that EMMPRIN is an inducer of MMP-9.

The significant correlations found between both MMP-9 and EMMPRIN, and myocardial injury assessed by biomarkers as well as infarct size measured by SPECT, have been sparsely explored in humans. In experimental AMI, MMP9 has been shown to increase infarct size and left ventricular fibrosis [13], in accordance with our findings.

An association between EMMPRIN and the degree of myocardial injury and LVEF has previously been reported in the work by Nie et al. [35], but this was a post mortem immunohistochemistry study which showed a strong increase in EMMPRIN around the zone of necrosis in the AMI group. This can to some degree be compared to our findings of the correlations between MMPs and biomarkers of AMI and also to our results of an inverse correlation between EMMPRIN and LVEF. An additional contribution to this understanding has been demonstrated in $\mathrm{CD} 147^{+/-}$ mice, where the disruption of the EMMPRIN-Cyclophilin A interaction reduced infarct size [36]. Our findings contribute to the suggestion that the expression of EMMPRIN is a decisive factor in regulating MMP-9 activity and thereby involved in myocardial remodeling.

The strength of the present study is the randomized design, the rather frequent sample collection for determination of the profiles, and gene expression analysis in a relatively large subpopulation. All patients were medically treated according to current guidelines; thus any influence by medication on the measured variables would therefore be equal in the randomized groups.

A limitation is that there was no bone marrow aspiration in the control group. There was, however, no significant difference between the two groups at baseline, that is, after the bone marrow aspiration in the mBMC group. It should be noted that the measures of circulating MMP-9 and TIMP-1 were performed in serum, as also used in many other studies. The proteins are released upon platelet activation during clotting, thus, the levels measured do not reflect the "true" circulating levels. However, the same standardized procedure for serum preparation was applied throughout the study, and therefore we assume that the comparisons between the randomized groups have been limited influenced. Confirmation of our results based on plasma samples is warranted. From the literature there are both coincide results and not when using plasma or serum samples, thus, it is important to take this into account when comparing results between studies. The gene expression analyses were performed in whole blood with circulating leukocytes as the RNA source, which might not be the most important source of either MMP-9 or EMMPRIN.

When performing stem cell transplantation as treatment regimen, several studies have discussed the timing, type of stem cells, and the procedure of the transplantation for optimization of the results $[37,38]$, but a conclusion has not yet been made.

\section{Conclusions}

Limited effects of intra coronary injection of mBMC transplantation on circulating levels as well as gene expression of MMP-9 and EMMPRIN in patients with STEMI treated with PCI could be demonstrated. EMMPRIN levels were reduced in both groups, whereas MMP-9 showed increased levels in the mBMC group. Both MMP-9 and EMMPRIN were significantly correlated to myocardial injury and infarct size, indicating that the regulation of metalloproteinases is important in the process of an AMI. The results contribute to the understanding of the pathophysiology of metalloproteinases in AMI, but further investigations are needed regarding timing and type of stem cells.

\section{Conflict of Interests}

The authors declare that there is no conflict of interests regarding the publication of this paper.

\section{Authors' Contribution}

Eline Bredal Furenes contributed in development of the study protocol, laboratory analyses, statistics, preparation, and discussion of paper. Trine Baur Opstad contributed to laboratory analyses, statistics, evaluation of results, and discussion of paper. Svein Solheim contributed in developing the study protocol, inclusion of patients, and discussion of paper. Ketil 
Lunde contributed in developing the study protocol, inclusion of patients, and discussion of paper. Harald Arnesen contributed in developing the study protocol, evaluation of study results, and discussion of paper. Ingebjørg Seljeflot contributed in developing the study protocol, evaluation of study results, and discussion of paper.

\section{Acknowledgments}

The study was supported by Stein Erik Hagens Foundation for Clinical Heart Research, Oslo, Norway. The authors thank Vibeke Bratseth for contribution to the laboratory analyses.

\section{References}

[1] J. M. Gaziano, "Global burden of cardiovascular disease," in Braunwald's Heart Disease, R. O. Bonow, D. P. Zipes, and P. Libby, Eds., pp. 1-19, Elsevier Saunders, Philadelphia, Pa, USA, 7th edition, 2005.

[2] P. Libby, P. M. Ridker, and A. Maseri, "Inflammation and atherosclerosis," Circulation, vol. 105, no. 9, pp. 1135-1143, 2002.

[3] F. S. Apple, A. H. A. Wu, J. Mair et al., "Future biomarkers for detection of ischemia and risk stratification in acute coronary syndrome," Clinical Chemistry, vol. 51, no. 5, pp. 810-824, 2005.

[4] D. Orlic, J. Kajstura, S. Chimenti et al., "Bone marrow cells regenerate infarcted myocardium," Nature, vol. 410, no. 6829, pp. 701-705, 2001.

[5] C. Peng, K. Yang, P. Xiang et al., "Effect of transplantation with autologous bone marrow stem cells on acute myocardial infarction," International Journal of Cardiology, vol. 162, no. 3, pp. 158-165, 2013.

[6] A. Flynn, X. Chen, E. O'Connell, and T. O'Brien, “A comparison of the efficacy of transplantation of bone marrow-derived mesenchymal stem cells and unrestricted somatic stem cells on outcome after acute myocardial infarction," Stem Cell Research and Therapy, vol. 3, no. 5, article 36, 2012.

[7] J.-H. Choi, J. Choi, W.-S. Lee et al., "Lack of additional benefit of intracoronary transplantation of autologous peripheral blood stem cell in patients with acute myocardial infarction," Circulation Journal, vol. 71, no. 4, pp. 486-494, 2007.

[8] L. Zhan-quan, Z. Ming, J. Yuan-zhe et al., "The clinical study of autologous peripheral blood stem cell transplantation by intracoronory infusion in patients with acute myocardial infarction (AMI)," International Journal of Cardiology, vol. 115, no. 1, pp. 52-56, 2007.

[9] S. Janssens, C. Dubois, J. Bogaert et al., "Autologous bone marrow-derived stem-cell transfer in patients with ST-segment elevation myocardial infarction: double-blind, randomised controlled trial," The Lancet, vol. 367, no. 9505, pp. 113-121, 2006.

[10] K. Lunde, S. Solheim, S. Aakhus et al., "Intracoronary injection of mononuclear bone marrow cells in acute myocardial infarction," The New England Journal of Medicine, vol. 355, no. 12, pp. 1199-1209, 2006.

[11] H. K. Grøgaard, O. E. Sigurjonsson, M. Brekke et al., "Cardiac accumulation of bone marrow mononuclear progenitor cells after intracoronary or intravenous injection in pigs subjected to acute myocardial infarction with subsequent reperfusion," Cardiovascular Revascularization Medicine, vol. 8, no. 1, pp. 2127, 2007.
[12] S. Dimmeler, A. M. Zeiher, and M. D. Schneider, "Unchain my heart: the scientific foundations of cardiac repair," The Journal of Clinical Investigation, vol. 115, no. 3, pp. 572-583, 2005.

[13] D. Kelly, G. Cockerill, L. L. Ng et al., "Plasma matrix metalloproteinase- 9 and left ventricular remodelling after acute myocardial infarction in man: a prospective cohort study," European Heart Journal, vol. 28, no. 6, pp. 711-718, 2007.

[14] M. Cheng, S. Hashmi, X. Mao, and Q. T. Zeng, "Relationships of adiponectin and matrix metalloproteinase-9 to tissue inhibitor of metalloproteinase-1 ratio with coronary plaque morphology in patients with acute coronary syndrome," Canadian Journal of Cardiology, vol. 24, no. 5, pp. 385-390, 2008.

[15] M. H. Tayebjee, S. Nadar, A. D. Blann, D. Gareth Beevers, R. J. MacFadyen, and G. Y. H. Lip, "Matrix metalloproteinase-9 and tissue inhibitor of metalloproteinase- 1 in hypertension and their relationship to cardiovascular risk and treatment: a substudy of the Anglo-Scandinavian Cardiac Outcomes Trial (ASCOT)," The American Journal of Hypertension, vol. 17, no. 9, pp. 764-769, 2004.

[16] E. B. Furenes, I. Seljeflot, S. Solheim, E. M. Hjerkinn, and $\mathrm{H}$. Arnesen, "Long-term influence of diet and/or omega-3 fatty acids on matrix metalloproteinase-9 and pregnancy-associated plasma protein-A in men at high risk of coronary heart disease," Scandinavian Journal of Clinical \& Laboratory Investigation, vol. 68, no. 3, pp. 177-184, 2008.

[17] R. Visse and H. Nagase, "Matrix metalloproteinases and tissue inhibitors of metalloproteinases: structure, function, and biochemistry," Circulation Research, vol. 92, no. 8, pp. 827-839, 2003.

[18] T. Sameshima, K. Nabeshima, B. P. Toole et al., "Glioma cell extracellular matrix metalloproteinase inducer (EMMPRIN) (CD147) stimulates production of membrane-type matrix metalloproteinases and activated gelatinase A in co-cultures with brain-derived fibroblasts," Cancer Letters, vol. 157, no. 2, pp. 177$184,2000$.

[19] C. Biswas, "Tumor cell stimulation of collagenase production by fibroblasts," Biochemical and Biophysical Research Communications, vol. 109, no. 3, pp. 1026-1034, 1982.

[20] P. Seizer and E. May, "Platelets and matrix metalloproteinases," Thrombosis \& Haemostasis, vol. 110, no. 5, pp. 903-909, 2013.

[21] K. Lunde, S. Solheim, S. Aakhus, H. Arnesen, M. Abdelnoor, and K. Forfang, "Autologous stem cell transplantation in acute myocardial infarction: the ASTAMI randomized controlled trial. Intracoronary transplantation of autologous mononuclear bone marrow cells, study design and safety aspects," Scandinavian Cardiovascular Journal, vol. 39, no. 3, pp. 150-158, 2005.

[22] K. J. Livak and T. D. Schmittgen, "Analysis of relative gene expression data using real-time quantitative PCR and the $2^{-\Delta \Delta C_{\mathrm{T}}}$ method," Methods, vol. 25, no. 4, pp. 402-408, 2001.

[23] C. Mias, O. Lairez, E. Trouche et al., "Mesenchymal stem cells promote matrix metalloproteinase secretion by cardiac fibroblasts and reduce cardiac ventricular fibrosis after myocardial infarction," Stem Cells, vol. 27, no. 11, pp. 2734-2743, 2009.

[24] T. Shu, B. Zeng, X. Ren, and Y. Li, "HO-1 modified mesenchymal stem cells modulate MMPs/TIMPs system and adverse remodeling in infarcted myocardium," Tissue and Cell, vol. 42, no. 4, pp. 217-222, 2010.

[25] N. Beyer Nardi and L. Da Silva Meirelles, "Mesenchymal stem cells: Isolation, in vitro expansion and characterization," Handbook of Experimental Pharmacology, vol. 174, pp. 249-282, 2006. 
[26] M. Roderfeld, T. Rath, S. Pasupuleti et al., "Bone marrow transplantation improves hepatic fibrosis in $\mathrm{Abcb}^{4-/-}$ mice via Th1 response and matrix metalloproteinase activity," Gut, vol. 61, no. 6, pp. 907-916, 2012.

[27] E. B. Furenes, H. Arnesen, S. Solheim, H. K. Grøgaard, P. Hoffmann, and I. Seljeflot, "The profile of circulating metalloproteinases after PCI in patients with acute myocardial infarction or stable angina," Thrombosis Research, vol. 124, no. 5, pp. 560-564, 2009.

[28] Y. Blanco, A. Saiz, E. Carreras, and F. Graus, "Changes of matrix metalloproteinase-9 and its tissue inhibitor (TIMP-1) after autologous hematopoietic stem cell transplantation in multiple sclerosis," Journal of Neuroimmunology, vol. 153, no. 12, pp. 190-194, 2004.

[29] T. Miyamoto, T. Muneta, T. Tabuchi et al., "Intradiscal transplantation of synovial mesenchymal stem cells prevents intervertebral disc degeneration through suppression of matrix metalloproteinase-related genes in nucleus pulposus cells in rabbits," Arthritis Research and Therapy, vol. 12, no. 6, article R206, 2010

[30] K. Tagami, T. Yujiri, T. Takahashi et al., "Increased serum levels of matrix metalloproteinase-9 in acute graft-versus-host disease after allogeneic haematopoietic stem cell transplantation," International Journal of Hematology, vol. 90, no. 2, pp. 248-252, 2009.

[31] Y. Wang, X. Hu, X. Xie, A. He, X. Liu, and J.-A. Wang, "Effects of mesenchymal stem cells on matrix metalloproteinase synthesis in cardiac fibroblasts," Experimental Biology and Medicine, vol. 236, no. 10, pp. 1197-1204, 2011.

[32] C. H. Yoon, J. Hur, K. W. Park, and et al, "Synergistic neovascularization by mixed transplantation of early endothelial progenitor cells and late outgrowth endothelial cells: The role of angiogenic cytokines and matrix metalloproteinases," Circulation, vol. 112, no. 11, pp. 1618-1627, 2005.

[33] Y. Ding, D. Xu, G. Feng, A. Bushell, R. J. Muschel, and K. J. Wood, "Mesenchymal stem cells prevent the rejection of fully allogenic islet grafts by the immunosuppressive activity of matrix metalloproteinase-2 and -9," Diabetes, vol. 58, no. 8, pp. 1797-1806, 2009.

[34] S. Xu, L. Tang, Y. Mi et al., "Clinical significance of leukotriene B4 and extracellular matrix metalloproteinase inducer in acute coronary syndrome," Clinical and Investigative Medicine, vol. 36, no. 6, pp. E282-E289, 2013.

[35] R. Nie, S. Xie, B. Du, X. Liu, B. Deng, and J. Wang, "Extracellular matrix metalloproteinase inducer (EMMPRIN) is increased in human left ventricle after acute myocardial infarction," Archives of Medical Research, vol. 40, no. 7, pp. 605-611, 2009.

[36] P. Seizer, C. Ochmann, T. Schönberger et al., "Disrupting the EMMPRIN (CD147)-cyclophilin a interaction reduces infarct size and preserves systolic function after myocardial ischemia and reperfusion," Arteriosclerosis, Thrombosis, and Vascular Biology, vol. 31, no. 6, pp. 1377-1386, 2011.

[37] M. Gnecchi, H. He, O. D. Liang et al., "Paracrine action accounts for marked protection of ischemic heart by Akt-modified mesenchymal stem cells," Nature Medicine, vol. 11, no. 4, pp. 367-368, 2005.

[38] F. H. Seeger, T. Tonn, N. Krzossok, A. M. Zeiher, and S. Dimmeler, "Cell isolation procedures matter: a comparison of different isolation protocols of bone marrow mononuclear cells used for cell therapy in patients with acute myocardial infarction," European Heart Journal, vol. 28, no. 6, pp. 766-772, 2007. 


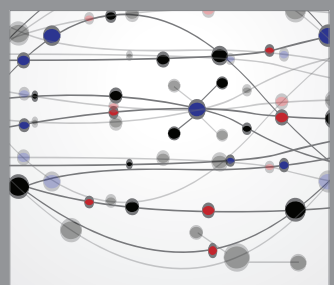

The Scientific World Journal
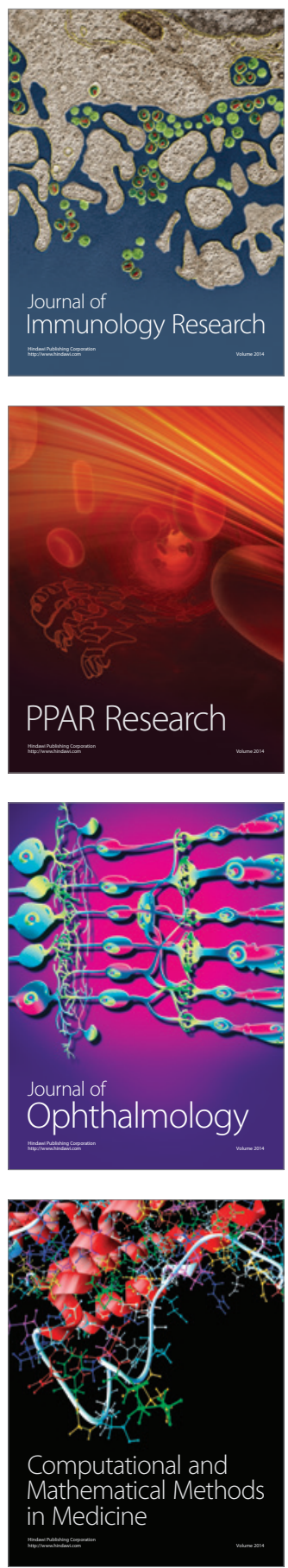

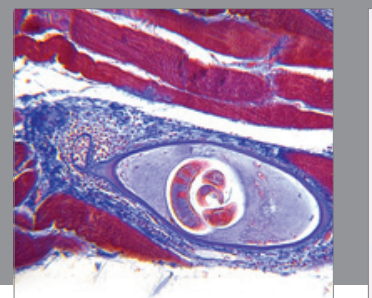

Gastroenterology

Research and Practice
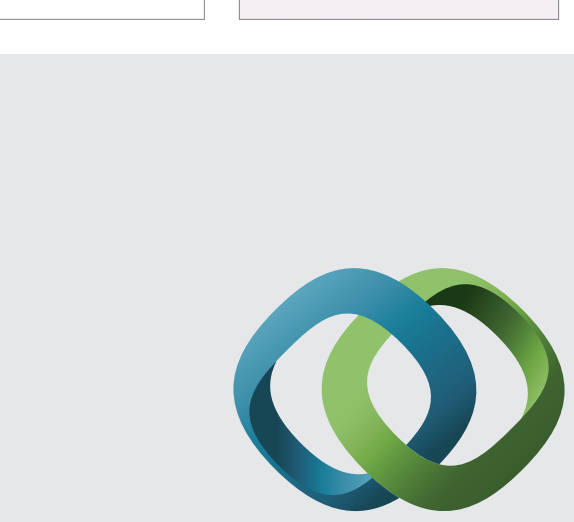

\section{Hindawi}

Submit your manuscripts at

http://www.hindawi.com
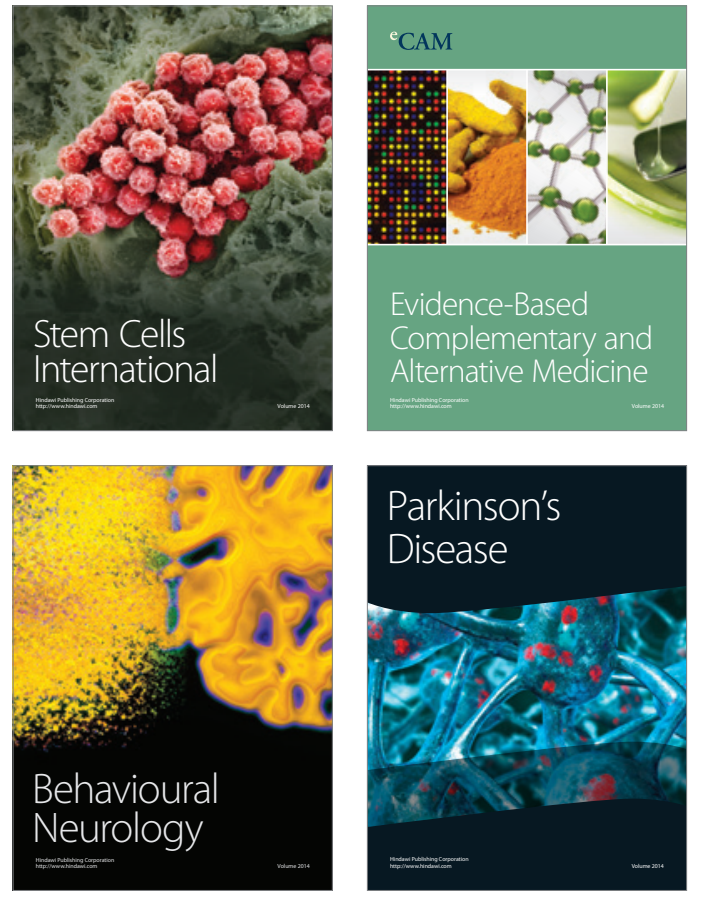
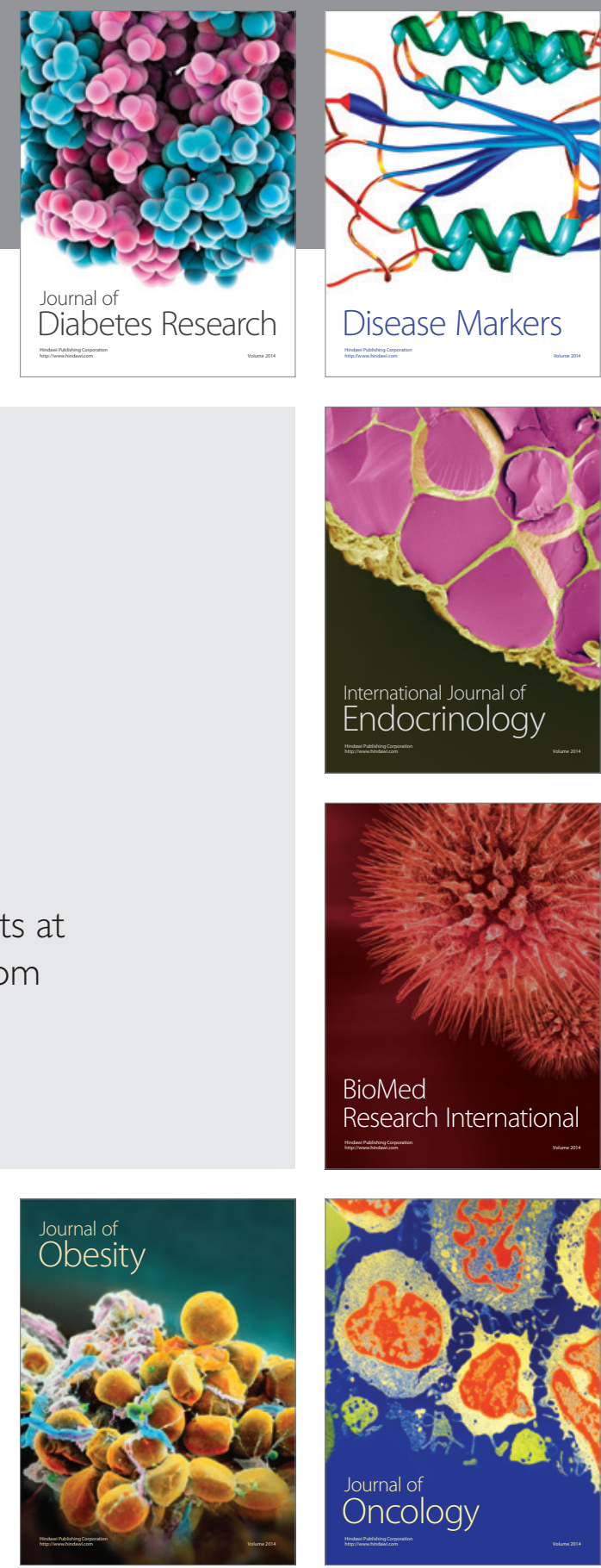

Disease Markers
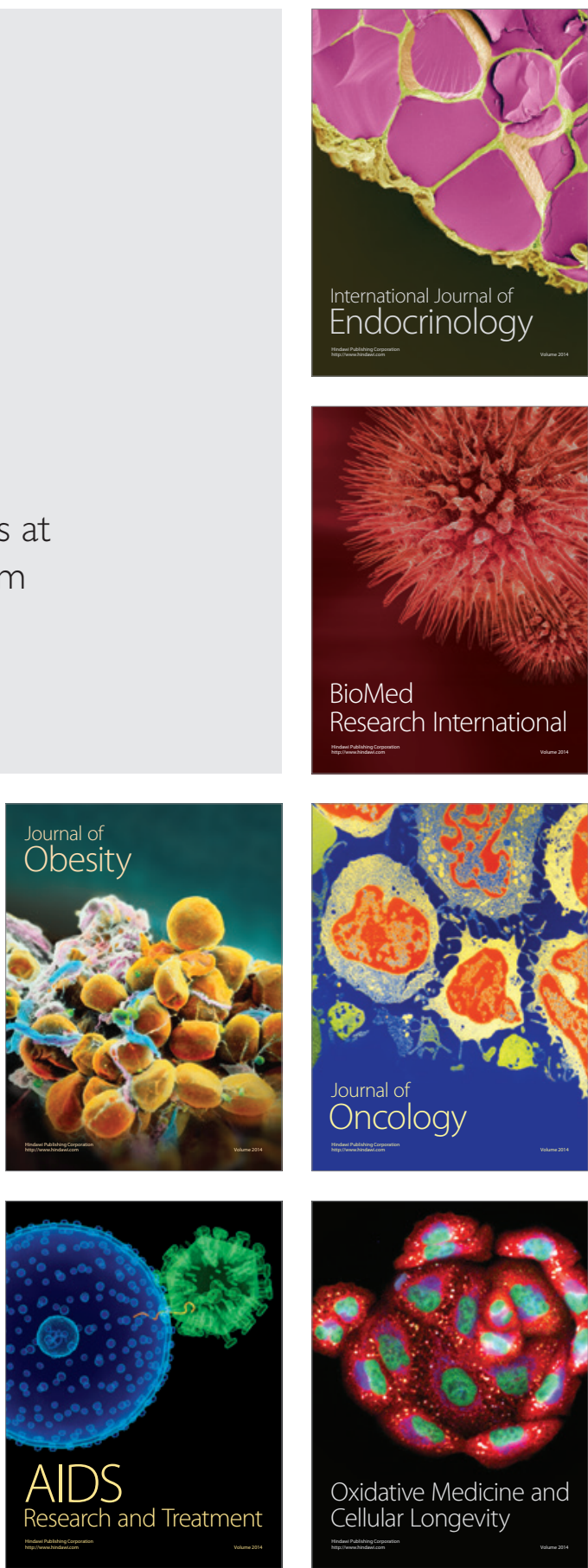